\title{
Pulse Wave Velocity in Korean American Men and Women
}

\author{
Jeongok G. Logan, PhD, RN [Project Manager] and \\ School of Nursing, University of North Carolina at Chapel Hill \\ Debra J. Barksdale, PhD, FNP-BC, FAANP [Associate Professor] \\ School of Nursing, University of North Carolina at Chapel Hill
}

\begin{abstract}
Arterial stiffness is an important clinical marker of cardiovascular diseases. Although many studies have been conducted on different racial groups, less is known about arterial stiffness in Asian Americans. Korean Americans constitute the fifth largest subgroup in the Asian American population and reportedly have a noticeably high prevalence of hypertension. The aims of this study were to assess arterial stiffness and blood pressure and to examine the effect of age and gender on arterial stiffness and blood pressure in 102 Korean American men and women aged 21 to 60 years. The values of arterial stiffness for Korean Americans in this study were compared to published reference values for other racial and ethnic groups. Arterial stiffness was measured by carotid-femoral pulse wave velocity, which is the gold standard for determining arterial stiffness. Findings indicated that aging was an important determinant of arterial stiffness, which increased linearly with age. Although there was no gender difference observed in arterial stiffness, the effect of age on arterial stiffness was greater in women than in men. After adjusting for covariates including age, body mass index, and smoking, multiple regression models showed that arterial stiffness and gender were significant predictors of systolic and diastolic blood pressure. The comparisons of these findings to those from several other studies that used the same method to measure arterial stiffness showed that Korean Americans may have levels of arterial stiffness that are similar to or slightly higher than those of other racial groups. Considering that arterial stiffness is an independent predictor of future development of hypertension, more studies are required to examine cardiovascular risk of this understudied group.
\end{abstract}

\section{Keywords}

arterial stiffness; hypertension; Korean Americans

\section{Background and Purpose}

Arterial stiffness is both an independent predictor of hypertension and an important clinical marker of cardiovascular diseases (CVDs). ${ }^{1,2}$ Arterial stiffness refers to the hardening of arterial walls and is caused mainly by decreased elastin and increased collagen fibers in the medial layer of arterial walls. ${ }^{3}$ Like other physiological processes, the extent of arterial stiffness can range from slight and insignificant to severe. Stiff arteries are, by nature, less compliant and therefore less distensible. Because pulse waves travel faster in stiffer arteries, the degree of stiffness can be determined by measuring arterial pulse wave velocity (PWV). Pulse wave velocity is considered the best method of measuring arterial stiffness because it

Copyright $\odot 2013$ Wolters Kluwer Health | Lippincott Williams \& Wilkins

Correspondence: Jeongok G. Logan, PhD, RN, School of Nursing, University of North Carolina at Chapel Hill, Carrington CB 7460, Chapel Hill, NC 27599-7460 (gang@email.unc.edu).

The authors have no conflicts of interest to disclose. 
is simple and reliable and can be easily reproduced. ${ }^{4}$ Pulse wave velocity is calculated from the distance the pulse travels between 2 arterial sites divided by pulse transit time: $\mathrm{PWV}=D$ (meters) $/ \Delta t$ (second). ${ }^{5}$ Carotid-femoral PWV (cfPWV) is currently accepted as the goldstandard measurement for arterial stiffness because it measures along the aortic and aortoiliac pathway, ${ }^{5}$ and the thoracic aorta and the abdominal aorta make the largest contribution to the arterial buffering function. ${ }^{6}$

Although arterial stiffness is implicated in the pathological processes of CVDs, less is known about the degree and extent of arterial stiffness in Asian Americans, particularly Korean Americans. A few studies have found racial difference in arterial stiffness. For example, in one study comparing 20 healthy young South Asian men with 20 age-matched healthy European white men, the South Asian men had markedly greater levels of arterial stiffness than did the European white men. ${ }^{7}$ The Multi-Ethnic Study of Atherosclerosis explored aortic distensibility in 4 ethnic groups (non-Hispanic white, African American, Hispanic, and Chinese) and reported that African Americans had the least aortic distensibility (indicating greater arterial stiffness), but aortic distensibility of the Chinese participants was not reported. ${ }^{8}$

Asian Americans are the fastest growing ethnic minority groups in the United States, and Korean Americans constitute the fifth largest subgroup in the Asian American population. ${ }^{9}$ Korean Americans reportedly have a noticeably high prevalence of hypertension. ${ }^{10}$ Nevertheless, studies to evaluate cardiovascular risk in Korean Americans are very limited, and we have found no published study to date that assessed arterial stiffness in Korean Americans. The aims of the study were to assess arterial stiffness and blood pressure (BP) in Korean Americans and to examine the effect of age and gender on arterial stiffness and BP in Korean American men and women. Furthermore, the values of arterial stiffness for Korean Americans in this study were compared to published reference values for other racial and ethnic groups.

\section{Methods}

Participants

Korean Americans were defined as people who were born in Korea and had lived in the United States for at least 1 year. After institutional review board approval was obtained, a total of 102 male and female Korean Americans aged 21 to 60 years in North Carolina were enrolled for the study.

Sample size was determined by power analysis. People who were pregnant or who had known cardiac conditions (eg, pacemaker, heart failure, coronary artery disease, myocardial infarction, left ventricular dysfunction, or valvular heart diseases), vascular conditions (eg, peripheral vascular diseases), or diabetes were excluded because these conditions may cause changes in arterial stiffness. People who were taking antihypertensive medications were also excluded because these drugs may cause muscular artery dilation that results in decreased PWV. ${ }^{11}$

\section{Variables and Their Measures}

Age, gender, and smoking were self-reported. Height was measured with a portable stadiometer and weight was measured with a portable electronic scale. Body mass index (BMI) was calculated by dividing weight by squared height $\left(\mathrm{kg} / \mathrm{m}^{2}\right)$.

Peripheral brachial BP was measured using a Welch Allyn Vital Signs Monitor 300 Series (Skaneateles Falls, New York). Because incorrect cuff size causes errors in BP measurement (cuff too large reads low and cuff too small reads high), ${ }^{12}$ the midsection circumference of 
the nondominant upper arm was obtained with a tape measure, and the proper sized cuff was selected according to the upper arm circumference. Using this automated BP monitoring device, BP was measured twice in the supine position, with a 2-minute rest between measures. ${ }^{13}$ The mean BP measurement was entered into the SphygmoCor software per protocol.

Carotid-femoral PWV was obtained noninvasively using the commercially available SphygmoCor system (AtCor Medical, Australia). Each carotid and femoral waveform was acquired by applying a pressure-sensitive transducer (tonometer) on the carotid and femoral sites. The transit time of the pulse from the left ventricle to the carotid artery $\left(t_{1}\right)$ and the transit time of the pulse from the left ventricle to the femoral artery $\left(t_{2}\right)$ were calculated by the system's software on the basis of electrocardiogram, using the foot-to-foot method. The distance from the suprasternal notch to the carotid artery site $\left(d_{\text {carotid }}\right)$ and the distance from the suprasternal notch to the femoral artery site ( $\left.d_{\text {femoral }}\right)$ were measured on the body using a tape measure. Carotid-femoral PWV is the difference in the distances from the suprasternal notch to 2 arterial sites $\left(d_{\text {pwv }}\right)$ divided by the time difference $(\Delta t)$ (Figure 1). ${ }^{14}$

\section{Procedures}

Data were collected in participants' home between 7 and 11 AM to standardize participant conditions; study procedures measuring arterial stiffness complied with the European Society of Cardiology's recommendations. ${ }^{5}$ All participants were asked to refrain from consuming coffee, smoking, and eating for at least 3 hours and drinking alcohol for 10 hours before data collection. ${ }^{5}$ Participants rested at least 10 minutes in the recumbent position before BP and arterial stiffness were measured. The participants were not spoken to and were asked not to speak during BP and arterial stiffness measurement. ${ }^{15}$ Investigators who were matched to the participant's gender and were well trained in using the SphygmoCor device measured cfPWV.

\section{Statistical Analysis}

All analyses were performed with SAS (version 9.2 for Windows). Data were tested for normality of distribution (skewness and kurtosis). Differences by gender in outcome variables were assessed by independent-samples $t$ test. The relationships between physiological variables and age were examined by bivariate correlation, and the effects of age on physiological variables were assessed by simple regression. Multiple regressions accompanied with collinearity diagnostics were used to identify predictors of arterial stiffness and BP. Statistical significance was inferred at a 2 -sided probability value less than .05 .

\section{Results}

The characteristics of participants and differences by gender are summarized in Table 1 . The mean (SD) age was $39.64(9.89)$ years. The mean (SD) BMI was $23.59(3.26) \mathrm{kg} / \mathrm{m}^{2}$, with 19 men (44\%) and 16 women (25\%) being overweight (BMI $\geq 25 \mathrm{~kg} / \mathrm{m}^{2}$ ). Of the 102 participants, 5 men $(12.50 \%)$ and 1 woman $(1.69 \%)$ were currently smoking. Seventy-eight participants $(76 \%)$ had college-level or higher education. Sixty-seven participants (66\%) reported that their household income were less than $\$ 49,999$ per year.

The cfPWV data were normally distributed, with skewness of 0.58 and kurtosis of -0.02 . The mean values of cfPWV in different age and gender groups are summarized in Table 2. Under age 40 years, men had higher cfPWV than women did, whereas above age 40 years, women had higher cfPWV than men did. However, for all age groups, the gender differences in cfPWV did not reach statistical significance. 
The result of bivariate correlation showed that age was highly correlated with cfPWV ( $r=$ $0.61, P<.0001)$ (Table 3). The simple linear regression for all participants showed that as age increased by a year, cfPWV increased by $0.07 \mathrm{~m} / \mathrm{s}\left(R^{2}=0.37, P<.0001\right)$. Women had higher age-associated increases in cfPWV than men did (Figure 2).

Because systolic BP (SBP) and diastolic BP (DBP) were highly correlated $(r=0.81, P<$. 0001) (Table 3), causing a collinearity issue, mean arterial pressure (MAP) was used to conduct multiple regressions to identify significant predictors of cfPWV. Age and MAP were identified as the major predictors of cfPWV in multiple regressions after controlling gender, BMI, and smoking status (Table 4).

Multiple regressions on SBP and DBP showed that cfPWV and gender, but not age, were significant predictors of SBP and DBP, after controlling BMI and smoking (Table 5 and 6). The results of tolerance and variance inflation factor and standard errors of the regression coefficients showed that correlation between age and cfPWV should not cause concern for collinearity in these models.

\section{Discussion}

Many studies have reported levels of arterial stiffness in various racial groups. $7,8,16$

However, the extent to which meaningful comparisons can be made between racial groups is unclear. Several different algorithms were used in different studies to calculate PWV. Furthermore, PWV was measured in different arterial sites, producing incomparable reference values. For example, some studies for Koreans in Korea measured arterial stiffness at the brachial and tibial arterial sites. ${ }^{17-19}$ The problem in comparing these findings to those of other studies using carotid and femoral arterial sites is that the values of brachial-ankle PWV will be higher than the cfPWV because there is increasing arterial stiffness moving from the heart to the periphery. ${ }^{20}$ Therefore, caution should be used when directly comparing levels of arterial stiffness between different studies. We will discuss our findings in relationship to those of other studies that have used the same measurement technique to obtain cfPWV as was used in this study.

In 2010, a population-based study $(\mathrm{N}=11,092)$ including 8 European countries reported reference values for cfPWV. ${ }^{21}$ In that study, cfPWV was measured by both SphygmoCor system and Complior system; thus, 2 different algorithms were used to detect the foot of the wave and to measure distance between the carotid and femoral arterial sites. Because the algorithm using the point of maximal systolic upstroke as the foot of wave and the direct measurement of distance between 2 arterial sites are known to overestimate cfPWV values, ${ }^{14,22}$ multiple equations were used to standardize the cfPWV values. The reported reference values for cfPWV based on age were 6.2 (age <30 years), 6.5 (age 30-39 years), 7.2 (age 40-49 years), 8.3 (age 50-59 years), 10.3 (age 60-69 years), and 10.9 (age $\geq 70$ years). ${ }^{21}$ Although our study population was different, we found similar values in Korean Americans between the ages of 21 and 60 years (those $>60$ years were not included in our study). Our findings suggest that Korean Americans might have similar degrees of arterial stiffness as Europeans do; however, reference values for Korean Americans could not be provided because of the small sample size $(n=102)$.

Another population-based study measured cfPWV using the SphygmoCor system in 998 healthy participants (92\% whites, $4 \%$ unspecified Asians) aged 18 to 90 years. ${ }^{23}$ According to the regression equation from that study, 40-year-old men and women are expected to have cfPWV values of 6.41 and $6.12 \mathrm{~m} / \mathrm{s}$, respectively. Therefore, the cfPWV of 7.11 and $6.91 \mathrm{~m} /$ $\mathrm{s}$ for Korean American men and women, respectively, in the present study seem to be somewhat higher than the levels of cfPWV for people in that study. 
Libhaber et $\mathrm{al}^{24}$ assessed cfPWV using the SphygmoCor in 305 South Africans of African ancestry (mean [SD] age, 39 [17] years) who had prevalent cardiovascular risk factors (24\% of the sample was hypertensive and $17 \%$ had diabetes mellitus). They reported that the mean (SD) cfPWV was $6.5(3.2) \mathrm{m} / \mathrm{s}$ for men $(\mathrm{n}=107$; mean [SD] age, 40 [18] years) and 6.0 (2.1) $\mathrm{m} / \mathrm{s}$ for women ( $\mathrm{n}=146$; mean [SD] age, 38 [16] years). In the present study, Korean Americans (who were free from known CVDs including hypertension and diabetes) had higher cfPWV and, therefore, stiffer arteries than did South Africans of African ancestry.

Although we compared our findings to those of other studies that used the same measurement method, the results of these comparisons should be considered tentative because of potential confounding factors. For example, BP is an important factor that influences the measurement of arterial stiffness. Ferreira et $\mathrm{al}^{25}$ suggested that there is a pressure-dependent difference of aortic stiffness in different racial groups, and therefore, it is important to consider BP when comparing arterial stiffness.

Because most population-based studies have been conducted with whites, more studies are recommended to confirm reference ranges in minority populations such as Korean Americans and to compare arterial stiffness between different racial and ethnic groups.

\section{Age-Associated Changes in Carotid-Femoral Pulse Wave Velocity}

Age is a major determinant of arterial stiffness. ${ }^{26}$ As age increases, changes such as thinning and fragmentation of elastin and increase in collagen occur in the media of arterial walls, causing stiffness of large elastic arteries. ${ }^{3}$ These changes are known to be independent of atherosclerosis (a specific type of arterial stiffness) as measured by intima-media thickness. ${ }^{27}$ Shiburi et al ${ }^{28}$ measured arterial stiffness using the SphygmoCor system in 185 healthy South Africans of African ancestry (mean age, 33.5 years) and reported a linear relationship between age and cfPWV. The correlation coefficient $(r=0.60)$ and regression coefficient $(\beta=.07)$ reported by their study were almost identical to those from this study of Korean Americans $(r=0.609$ and $\beta=.07)$.

Some studies with a population-based sample have reported that the age-related changes in cfPWV are nonlinear. For example, the Framingham Heart Study, with 521 healthy participants, reported that age was a significant predictor of cfPWV and that cfPWV increased more significantly in older individuals. In another study of 998 healthy individuals (majority white), there was also a stronger effect of age in participants older than 50 years. $^{23}$ The linear relationship between age and cfPWV observed in the present study may be due to the relatively young participants (mean [SD] age, 39.64 [9.89] years). Rogers et al ${ }^{29}$ measured cfPWV for 24 volunteers (mean [SD] age, 54 [15] years) and reported a correlation coefficient between age and cfPWV $(r=0.62)$, but their linear regression coefficient was higher $(\beta=.097$ ) than our finding. This relationship may be explained by the fact that the mean age of the 24 participants in that study was higher than that of the 102 Korean Americans in this study. In addition, their small sample size limits generalizability.

\section{Blood Pressure and Carotid-Femoral Pulse Wave Velocity}

In addition to age, MAP was also identified as a significant predictor of cfPWV, independent of age, gender, BMI, and smoking. As arterial BP increases, acute and reversible stiffening of large arteries occurs. Over time, elevated BP can lead to vascular remodeling, hypertrophy, and hyperplasia that can induce and accelerate arterial stiffening. ${ }^{0}, 31$ On the other hand, arterial stiffness may be a preexisting condition that contributes to the development of high BP. Thus, the relationship between arterial stiffness and hypertension is viewed as bidirectional. ${ }^{31}$ 
Although age has been identified as an important determinant of SBP in previously published studies, ${ }^{32,33}$ a recent population study that measured cfPWV in 449 normotensive or untreated hypertensive volunteers (mean age, 53 years) demonstrated that cfPWV was an independent determinant of the longitudinal increase in SBP after adjusting for covariates including age, BMI, and MAP. ${ }^{34}$ The regression results of the present study also showed that the effect of age on SBP did not remain significant when PWV was controlled. Diastolic BP continuously increases up to about 60 years of age and then starts to decrease after 60 years of age. ${ }^{32,35}$ Age was significantly and positively related to DBP in the bivariate correlation for this study, in which none were older than 60 years. The relationship between age and DBP did not also remain significant when PWV was controlled. The effect of age on BP reported in the previous studies may actually be, in part, the effect of age-associated increases and related effects of PWV on BP. Because our study was cross-sectional, longitudinal studies should be conducted to clarify and compare the age-associated increases of BP and the PWV-associated increases of BP.

\section{Gender Difference in Carotid-Femoral Pulse Wave Velocity}

Whether levels of arterial stiffness differ between men and women is not yet conclusive. No differences by gender in arterial stiffness were found in a study of 998 participants (age 1890 years). ${ }^{23}$ The Framingham Heart Study, with 521 participants (188 men with a mean [SD] age of 56 [9] years and 333 women with a mean [SD] age of 57 [8] years), also reported that gender differences in arterial stiffness were not significant. ${ }^{36}$ Likewise, no gender differences in arterial stiffness were found between the 41 men and the 61 women in our study of Korean Americans. On the other hand, findings from one study that measured PWV by whole-body impedance cardiography in 799 participants (age 25-76 years) reported that men had higher PWV values than did women in those younger than 60 years but similar levels of PWV in those older than 60 years. ${ }^{16}$

Although cfPWV was not different by gender in Korean Americans, age-associated increases in cfPWV were almost 2 times higher in women $(\beta=.086, P<.0001)$ than in men $(\beta=.047, P<.001)$. More dramatic increases in arterial stiffness in women when compared with men have consistently been observed in previous studies. ${ }^{16,23,36}$ This may explain the higher prevalence of CVDs in older women. ${ }^{37}$ Further studies are recommended to explore cardiovascular risks and arterial stiffness in older Korean American women.

\section{Limitations}

This study of Korean Americans has several potential limitations. First, because our study included only 102 Korean Americans, findings cannot be generalized to the larger Korean American population. Second, this study was cross-sectional in design. To confirm ageassociated changes in cardiovascular markers, longitudinal studies should be conducted. Third, this study did not control other classic risk factors potentially influencing arterial stiffness such as hyperlipidemia and exercise. To confirm the relationship of arterial stiffness to age, BP, and gender, further studies that control well-established cardiovascular risk factors should be conducted.

\section{Conclusion}

This study examined arterial stiffness in 102 Korean American men and women 21 to 60 years of age by measuring cfPWV using the SphygmoCor system. To our knowledge, this was the first study to evaluate arterial stiffness in Korean Americans. Findings indicated that age and BP were important determinants of arterial stiffness. There was no gender difference observed in arterial stiffness; however, the effect of age on arterial stiffness was greater in women than in men. After adjusting for covariates including BMI and smoking, 
arterial stiffness and gender, but not age, were significant and independent predictors of SBP and DBP in this study. The comparisons of these findings to those from several other studies that used the same method for determining arterial stiffness showed that these Korean Americans may have levels of arterial stiffness that are similar to or slightly higher than those of other racial/ethnic groups. Considering that arterial stiffness may be an independent predictor of future development of hypertension, more studies are required to examine cardiovascular risk of Korean Americans and to compare arterial stiffness of this understudied group with other racial groups.

\section{Acknowledgments}

This study was supported by a Ruth L. Kirschstein National Research Service Award (F31) from the National Institute of Nursing Research.

We also thank Dr Joanne Harrell, John Carlson, Dr Mi Ja Kim, Dr Barbara Carlson, and Dr Pamela Johnson Rowsey for supporting this study.

\section{References}

1. Cernes R, Zimlichman R, Shargorodsky M. Arterial elasticity in cardiovascular disease: focus on hypertension, metabolic syndrome and diabetes. Adv Cardiol. 2008; 45:65-81. [PubMed: 18230956]

2. Laurent S, Boutouyrie P. Arterial stiffness: a new surrogate end point for cardiovascular disease? J Nephrol. 2007; 20(suppl 12):S45-S50. [PubMed: 18050143]

3. Levy, MN.; Pappano, AJ.; Berne, RM. Cardiovascular Physiology. 9. Philadelphia, PA: Mosby/ Elsevier; 2007.

4. Mackenzie IS, Wilkinson IB, Cockcroft JR. Assessment of arterial stiffness in clinical practice. QJM. 2002; 95(2):67-74. [PubMed: 11861952]

5. Laurent S, Cockcroft J, Van Bortel L, et al. Expert consensus document on arterial stiffness: methodological issues and clinical applications. Eur Heart J. 2006; 27(21):2588-2605. [PubMed: 17000623]

6. Laurent S, Caviezel B, Beck L, et al. Carotid artery distensibility and distending pressure in hypertensive humans. Hypertension. 1994; 23(6 pt 2):878-883. [PubMed: 8206621]

7. Din JN, Ashman OA, Aftab SM, Jubb AW, Newby DE, Flapan AD. Increased arterial stiffness in healthy young South Asian men. J Hum Hypertens. 2006; 20(2):163-165. [PubMed: 16306999]

8. Malayeri AA, Natori S, Bahrami H, et al. Relation of aortic wall thickness and distensibility to cardiovascular risk factors (from the Multi-Ethnic Study of Atherosclerosis [MESA]). Am J Cardiol. 2008; 102(4):491-496. [PubMed: 18678312]

9. Reeves, T.; Bennett, CE. U.S. Census Bureau. . We the People: Asians in the United States. Washington, DC: U.S. Dept. of Commerce, Economic and Statistics Administration, U.S. Census Bureau; 2004. p. 24

10. Kim MT, Kim KB, Juon HS, Hill MN. Prevalence and factors associated with high blood pressure in Korean Americans. Ethnic Disparities. 2000; 10(3):364-374.

11. O'Rourke MF, Nichols WW, O'Brien E. Effects of ramipril on arterial stiffness. Hypertension. 2005; 46(4):e14. author reply e14-e15. [PubMed: 16195488]

12. Pickering TG, Hall JE, Appel LJ, et al. Recommendations for blood pressure measurement in humans and experimental animals, part 1: blood pressure measurement in humans: a statement for professionals from the Subcommittee of Professional and Public Education of the American Heart Association Council on High Blood Pressure Research. Hypertension. 2005; 45(1):142-161. [PubMed: 15611362]

13. Pickering TG, Hall JE, Appel LJ, et al. Recommendations for blood pressure measurement in humans and experimental animals, part 1: blood pressure measurement in humans: a statement for professionals from the Subcommittee of Professional and Public Education of the American Heart Association Council on High Blood Pressure Research. Circulation. 2005; 111(5):697-716. [PubMed: 15699287] 
14. Millasseau SC, Stewart AD, Patel SJ, Redwood SR, Chowienczyk PJ. Evaluation of carotidfemoral pulse wave velocity: influence of timing algorithm and heart rate. Hypertension. 2005; 45(2):222-226. [PubMed: 15642772]

15. Umana E, Ahmed W, Fraley MA, Alpert MA. Comparison of oscillometric and intraarterial systolic and diastolic blood pressures in lean, overweight, and obese patients. Angiology. 2006; 57(1):41-45. [PubMed: 16444455]

16. Koivistoinen T, Koobi T, Jula A, et al. Pulse wave velocity reference values in healthy adults aged 26-75 years. Clin Physiol Funct Imaging. 2007; 27(3):191-196. [PubMed: 17445071]

17. Nam HJ, Jung IH, Kim J, et al. Association between brachial-ankle pulse wave velocity and occult coronary artery disease detected by multi-detector computed tomography [published online ahead of print February 9, 2011]. Int J Cardiol. 2011

18. Kim JB, Kang WY, Kim SJ, et al. The impact of chronic cigarette smoking on arterial stiffness in Korea. J Korean Geriatr Soc. 2011; 15(1):47-52.

19. Park BJ, Lee HR, Shim JY, Lee JH, Jung DH, Lee YJ. Association between resting heart rate and arterial stiffness in Korean adults. Arch Cardiovasc Dis. 2010; 103(4):246-252. [PubMed: 20656635]

20. Tillin T, Chambers J, Malik I, et al. Measurement of pulse wave velocity: site matters. J Hypertens. 2007; 25(2):383-389. [PubMed: 17211245]

21. Reference Values for Arterial Stiffness' Collaboration. Determinants of pulse wave velocity in healthy people and in the presence of cardiovascular risk factors: "establishing normal and reference values”. Eur Heart J. 2010; 31(19):2338-2350. [PubMed: 20530030]

22. Rajzer MW, Wojciechowska W, Klocek M, Palka I, Brzozowska-Kiszka M, Kawecka-Jaszcz K. Comparison of aortic pulse wave velocity measured by three techniques: Complior, SphygmoCor and Arteriograph. J Hypertens. 2008; 26(10):2001-2007. [PubMed: 18806624]

23. McEniery CM, Yasmin, Hall IR, Qasem A, Wilkinson IB, Cockcroft JR. Normal vascular aging: differential effects on wave reflection and aortic pulse wave velocity: the Anglo-Cardiff Collaborative Trial (ACCT). J Am Coll Cardiol. 2005; 46(9):1753-1760. [PubMed: 16256881]

24. Libhaber E, Woodiwiss AJ, Libhaber C, et al. Gender-specific brachial artery blood pressureindependent relationship between pulse wave velocity and left ventricular mass index in a group of African ancestry. J Hypertens. 2008; 26(8):1619-1628. [PubMed: 18622241]

25. Ferreira AV, Viana MC, Mill JG, Asmar RG, Cunha RS. Racial differences in aortic stiffness in normotensive and hypertensive adults. J Hypertens. 1999; 17(5):631-637. [PubMed: 10403606]

26. Lee HY, Oh BH. Aging and arterial stiffness. Circ J. 2010; 74(11):2257-2262. [PubMed: 20962429]

27. Wykretowicz A, Gerstenberger P, Guzik P, et al. Arterial stiffness in relation to subclinical atherosclerosis. Eur J Clin Invest. 2009; 39(1):11-16. [PubMed: 19087126]

28. Shiburi CP, Staessen JA, Maseko M, et al. Reference values for SphygmoCor measurements in South Africans of African ancestry. Am J Hypertens. 2006; 19(1):40-46. [PubMed: 16461189]

29. Rogers WJ, Hu YL, Coast D, et al. Age-associated changes in regional aortic pulse wave velocity. J Am Coll Cardiol. 2001; 38(4):1123-1129. [PubMed: 11583892]

30. Risler NR, Cruzado MC, Miatello RM. Vascular remodeling in experimental hypertension. Sci World J. 2005; 5:959-971.

31. Franklin SS. Arterial stiffness and hypertension: a two-way street? Hypertension. 2005; 45(3):349351. [PubMed: 15710783]

32. Franklin SS, Gustin Wt, Wong ND, et al. Hemodynamic patterns of age-related changes in blood pressure. The Framingham Heart Study. Circulation. 1997; 96(1):308-315. [PubMed: 9236450]

33. Ko GT, Chan JC, Cockram CS. Age, body mass index and 2-hour plasma glucose are the major determinants of blood pressure in Chinese women newly diagnosed to have glucose intolerance. Int J Cardiol. 1999; 69(1):33-39. [PubMed: 10362370]

34. Najjar SS, Scuteri A, Shetty V, et al. Pulse wave velocity is an independent predictor of the longitudinal increase in systolic blood pressure and of incident hypertension in the Baltimore Longitudinal Study of Aging. J Am Coll Cardiol. 2008; 51(14):1377-1383. [PubMed: 18387440] 
35. Burt VL, Whelton P, Roccella EJ, et al. Prevalence of hypertension in the US adult population. Results from the Third National Health and Nutrition Examination Survey, 1988-1991. Hypertension. 1995; 25(3):305-313. [PubMed: 7875754]

36. Mitchell GF, Parise H, Benjamin EJ, et al. Changes in arterial stiffness and wave reflection with advancing age in healthy men and women: the Framingham Heart Study. Hypertension. 2004; 43(6):1239-1245. [PubMed: 15123572]

37. Rosamond W, Flegal K, Furie K, et al. Heart disease and stroke statistics-2008 update: a report from the American Heart Association Statistics Committee and Stroke Statistics Subcommittee. Circulation. 2008; 117(4):e25-e146. [PubMed: 18086926] 


\section{What's New and Important}

- This was the first study to evaluate arterial stiffness in Korean Americans.

- Findings indicated the following:

- Aging was an important determinant of arterial stiffness, which increased linearly with age.

- The effect of age on arterial stiffness was greater in women than in men.

- Arterial stiffness and gender, but not age, were significant and independent predictors of systolic blood pressure (SBP) and diastolic blood pressure.

- The comparisons of these findings to those from several other studies that used the same methods to measure arterial stiffness showed that Korean Americans may have levels of arterial stiffness that are similar to or slightly higher than those of whites and South Africans of African ancestry.

- Considering that arterial stiffness is an independent predictor of future development of hypertension, more studies are required to examine cardiovascular risk of this understudied group. 
t1: The transit time between the R wave of EKG and the foot of carotid artery wave form (A)

t2: The transit time between the R wave of EKG and the foot of femoral artery wave form (B)

$\Delta t$ : The mean difference in time between $A$ and $B$, taking the difference between $t 2$ and $t 1$

$d_{\text {carotid: }}$ : the distance from the suprasternal notch to the carotid artery site

$d_{\text {femoral }}$ : the distance from the suprasternal notch to the femoral artery site

$d p w v$ : The difference between $d_{\text {femoral }}$ and $d_{\text {carotid }}$

$$
P W V=\frac{d_{P W V}}{\overline{\Delta t}}(\mathrm{~m} / \mathrm{s})
$$

Carotid artery pulse wave (A)

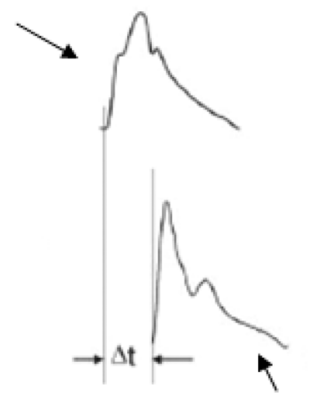

Femoral artery pulse wave (B)

FIGURE 1.

Pulse wave velocity (PWV) using SphygmoCor. Abbreviation: EKG, electrocardiogram. 


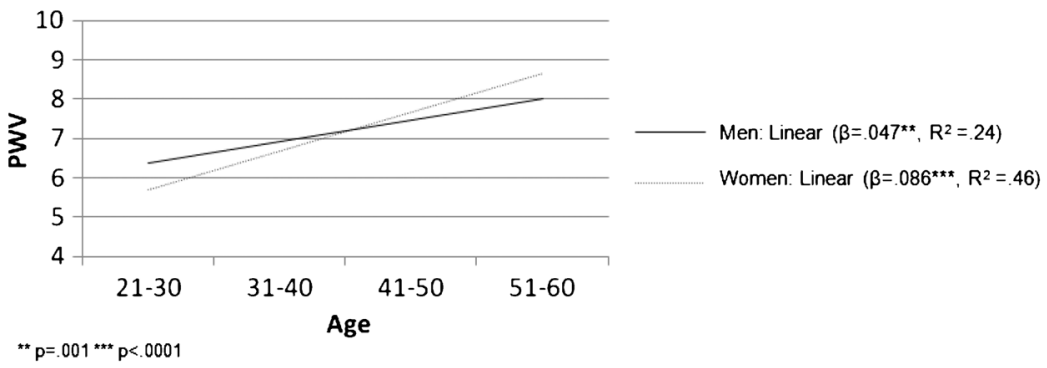

FIGURE 2.

Age-associated increase in pulse wave velocity (PWV) in men and women. 
TABLE 1

Characteristics of the Study Participants and Differences by Gender

\begin{tabular}{lcccc}
\hline Variable & All $(\mathbf{N}=\mathbf{1 0 2})$ & Men $(\mathbf{n}=\mathbf{4 1})$ & Women $(\mathbf{n}=\mathbf{6 1})$ & $\boldsymbol{P}$ \\
\hline Age, y & $39.64 \pm 9.89$ & $39.76 \pm 10.44$ & $38.96 \pm 9.71$ & .6884 \\
Body mass index, $\mathrm{kg} / \mathrm{m}^{2}$ & $23.59 \pm 3.26$ & $24.45 \pm 2.87$ & $23.00 \pm 3.39$ & .0272 \\
Current smoking status, yes & $6(5.9)$ & $5(12.50)$ & $1(1.69)$ & .058 \\
Peripheral systolic blood pressure, $\mathrm{mm} \mathrm{Hg}$ & $119.70 \pm 14.91$ & $125.82 \pm 12.74$ & $115.59 \pm 14.95$ & .0005 \\
Peripheral diastolic blood pressure, $\mathrm{mm} \mathrm{Hg}$ & $71.92 \pm 9.27$ & $75.82 \pm 8.19$ & $69.29 \pm 9.09$ & .0004 \\
Peripheral pulse pressure, $\mathrm{mm} \mathrm{Hg}$ & $47.78 \pm 9.23$ & $50.00 \pm 9.53$ & $46.29 \pm 8.79$ & .0464 \\
\hline
\end{tabular}

Values are mean \pm SD or frequency (\%). 


\section{TABLE 3}

Correlation Among Age, Pulse Wave Velocity (m/s), and Blood Pressure (BP)

\begin{tabular}{lllll}
\hline & Age & PWV & SBP & DBP \\
\hline Age & 1.000 & $0.609^{a}$ & $0.246^{b}$ & $0.392^{a}$ \\
PWV & & 1.000 & $0.449^{a}$ & $0.558^{a}$ \\
SBP & & & 1.000 & $0.807^{a}$ \\
DBP & & & & 1.00 \\
\hline
\end{tabular}

Abbreviations: DBP, diastolic BP; SBP, systolic BP; PWV, pulse wave velocity.

$\mathrm{N}=102$.

${ }_{P}^{a}<.0001$.

$b_{P<.05 .}$ 
